\title{
Stability and superstability of generalized quadratic ternary derivations on non- Archimedean ternary Banach algebras: a fixed point approach
}

\author{
Choonkil Park' Madjid Eshaghi Gordji ${ }^{2}$ and Yeol Je Cho ${ }^{3^{*}}$
}

\footnotetext{
* Correspondence: yjcho@gnu.ac.kr ${ }^{3}$ Department of Mathematics Education and the RINS, Gyeongsang National University, Chinju 660-701, Korea Full list of author information is available at the end of the article
}

\begin{abstract}
Using fixed point method, we prove the Hyers-Ulam stability and the superstability of generalized quadratic ternary derivations on non-Archimedean ternary Banach algebras. Indeed, we investigate the Hyers-Ulam stability and the superstability of the system of functional equations

$$
\left\{\begin{array}{l}
f([a b c])=\left[f(a) b^{2} c^{2}\right]+\left[a^{2} f(b) c^{2}\right]+\left[a^{2} b^{2} f(c)\right] \\
g([a b c])=\left[g(a) b^{2} c^{2}\right]+\left[a^{2} f(b) c^{2}\right]+\left[a^{2} b^{2} f(c)\right] \\
g(u x+v y)+g(u x-v y)=2 u^{2} g(x)+2 v^{2} g(y) \\
f(u x+v y)+f(u x-v y)=2 u^{2} f(x)+2 v^{2} f(y)
\end{array}\right.
$$

in non-Archimedean ternary Banach algebras.

Mathematics Subject Classification 2010: Primary 39B52; 47H10; 39B72; 46L57; 17B40; 13N15; 17A40; 20N10.
\end{abstract}

Keywords: Quadratic functional equation, quadratic derivation, superstability, nonArchimedean algebra, fixed point

\section{Introduction and preliminaries}

The stability problem of functional equations had been first raised by Ulam [1]. This problem solved by Hyers [2] in the framework of Banach spaces. In 1978, Th.M. Rassias [3] provided a generalization of the Hyers' theorem by proving the existence of unique linear mappings near approximate additive mappings. Găvruta [4] obtained generalized result of the Th.M. Rassias' theorem which allows the Cauchy difference to be controlled by a general unbounded function.

Bourgin [5] proved the stability of ring homomorphisms between two unital Banach algebras and Badora [6] gave a generalization of the Bourgin result. The stability result concerning derivations between operator algebras was first obtained by Šemrl [7]. In [8], Badora proved the stability of functional equation

$$
f(x y)=x f(y)+f(x) y,
$$

(c) 2012 Park et al; licensee Springer. This is an Open Access article distributed under the terms of the Creative Commons Attribution License (http://creativecommons.org/licenses/by/2.0), which permits unrestricted use, distribution, and reproduction in any medium, provided the original work is properly cited. 
where $f$ is a mapping on normed algebra $A$ with the unit. Park et al. proved the stability of homomorphisms and derivations in Banach algebras, Banach ternary algebras, $C^{*}$-algebras, Lie $C^{*}$-algebras and $C^{*}$-ternary algebras (see [9-14]).

Let $\mathcal{A}$ be a ternary algebra. A mapping $f: \mathcal{A} \rightarrow \mathcal{A}$ is called a quadratic ternary derivation if $f$ is a quadratic mapping satisfies

$$
f([a, b, c])=\left[f(a), b^{2}, c^{2}\right]+\left[a^{2}, f(b), c^{2}\right]+\left[a^{2}, b^{2}, f(c)\right]
$$

for all $a, b, c \in \mathcal{A}$.

A mapping $g: \mathcal{A} \rightarrow \mathcal{A}$ is called a generalized quadratic ternary derivation if there exists a quadratic ternary derivation $f: \mathcal{A} \rightarrow \mathcal{A}$ such that

$$
g([a, b, c])=\left[g(a), b^{2}, c^{2}\right]+\left[a^{2}, f(b), c^{2}\right]+\left[a^{2}, b^{2}, f(c)\right]
$$

for all $a, b, c \in \mathcal{A}$.

Let $\mathbb{K}$ denote a field and function (valuation absolute) $|\cdot|$ from $\mathbb{K}$ into $[0, \infty)$. A non-Archimedean valuation is a function $|\cdot|$ that satisfies the strong triangle inequality, namely,

$$
|x+y| \leq \max \{|x|,|y|\} \leq|x|+|y|
$$

for all $x, y \in \mathbb{K}$. The associated field $\mathbb{K}$ is referred to as a non-Archimedean field. Clearly, $|1|=|-1|=1$ and $|n| \leq 1$ for all $n \geq 1$. A trivial example of a non-Archimedean valuation is the function $|\cdot|$ taking everything except 0 into 1 and $|0|=0$. We always assume in addition that $|\cdot|$ is non trivial, i.e., there exists $z \in \mathbb{K}$ such that $|z|$ $\neq 0,1$.

Let $X$ be a linear space over a field $\mathbb{K}$ with a non-Archimedean nontrivial valuation | . |. A function $\|\cdot\|: X \rightarrow[0, \infty)$ is said to be a non-Archimedean norm if it is a norm over $\mathbb{K}$ with the strong triangle inequality (ultrametric), namely,

$$
\|x+y\| \leq \max \{\|x\|,\|y\|\}
$$

for all $x, y \in X$. Then $(X,\|\cdot\|)$ is called a non-Archimedean space. In any such a space a sequence $\left\{x_{n}\right\}_{n \in \mathbb{N}}$ is a Cauchy sequence if and only if $\left\{x_{n+1}-x_{n}\right\}_{n \in \mathbb{N}}$ converges to zero. By a complete non-Archimedean space we mean one in which every Cauchy sequence is convergent.

A non-Archimedean ternary Banach algebra is a complete non-Archimedean space $\mathcal{A}$ equipped with a ternary product $(x, y, z) \rightarrow[x, y, z]$ of $\mathcal{A}^{3}$ into $\mathcal{A}$ which is $\mathcal{K}$-linear in each variables and associative in the sense that

$$
[x, y,[z, w, v]]=[x,[w, z, y], v]=[[x, y, z], w, v]
$$

and satisfies the following:

$$
\|[x, y, z]\| \leq\|x\| \cdot\|y\| \cdot\|z\|
$$

(see [15-19]).

Arriola and Beyer [20] initiated the stability of functional equations in non-Archimedean spaces. In fact, they established stability of Cauchy functional equations over $p$ adic fields. After their results some papers (see, for instance, [21-27]) on the stability of other equations in such spaces have been published. Although different methods are 
known for establishing the stability of functional equations, almost all proofs depend on Hyers' method in [2]. In 2003, Radu [28] employed the alternative fixed point theorem, due to Diaz and Margolis [29], to prove the stability of Cauchy additive functional equation. Subsequently, this method was applied to investigate the Hyers-Ulam stability for Jensen functional equation [30], as well as for the Cauchy functional equation [31], by considering a general control function $\phi(x, y)$, with suitable properties. Using such an elegant idea, several authors applied the method to investigate the stability of some functional equations (see [12,32-34]).

Recently, Eshaghi Gordji and Khodaei [35] proved the Hyers-Ulam stability of the following quadratic functional equation

$$
f(a x+b y)+f(a x-b y)=2 a^{2} f(x)+2 b^{2} f(y)
$$

for nonzero fixed integers $a, b$. Recently, Eshaghi Gordji and Alizadeh [36,37] proved the Hyers-Ulam stability of homomorphisms and derivations on non-Archimedean Banach algebras.

In this paper, by using fixed point method, we establish the stability of generalized quadratic ternary derivations related to the quadratic functional equation (1.1) over non-Archimedean ternary Banach algebras.

In 1897, Hensel [38] discovered the $p$-adic numbers as a number theoretical analogue of power series in complex analysis. During the last three decades $p$-adic numbers have gained the interest of physicists for their research, in particular, in the problems coming from quantum physics, $p$-adic strings and superstrings [39,40]. A key property of $p$-adic numbers is that they do not satisfy the Archimedean axiom: For any $x, y>0$, there exists $n \in \mathbb{N}$ such that $x<n y$ (see $[41,42]$ ).

\section{Main results}

Using the strong triangle inequality in the proof of the main result of [29], we get the following result:

Theorem 2.1. (Non-Archimedean Alternative Contraction Principle) Let $(\Omega, d)$ be a non-Archimedean generalized complete metric space and $T: \Omega \rightarrow \Omega$ a strictly contractive mapping (that is, $d(T(x), T(y)) \leq L d(x, y)$ for all $x, y \in T$ and a Lipschitz constant $L$ $<1)$. Let $x \in \Omega$. If either

(a) $d\left(T^{n}(x), T^{n+1}(x)\right)=\infty$ for all $n \geq 0$, or

(b) there exists some $n_{0} \geq 0$ such that $d\left(T^{n}(x), T^{n+1}(x)\right)<\infty$ for all $n \geq n_{0}$, then the sequence $\left\{T^{n}(x)\right\}$ is convergent to a unique fixed point $x^{*}$ of $T$ in the set

$$
\Lambda=\left\{y \in \Omega: d\left(T^{n_{0}}(x), y\right)<\infty\right\}
$$

and $d\left(y, x^{*}\right) \leq d(y, T(y))$ for all $y$ in this set.

From now on, we assume that $(\mathcal{A},[\cdot, \cdot, \cdot])$ is a non-Archimedean ternary Banach algebra and $\ell \in\{-1,1\}$ is fixed. Also, we suppose that $|4|<1$ and that $4 \neq 0$ in $\mathbb{K}$ (i.e., the characteristic of $\mathbb{K}$ is not 4$)$. We denote $[a, b, c]$ by $[a b c]$ in ternary Banach algebra $\mathcal{A}$.

Theorem 2.2. Let $g, f: \mathcal{A} \rightarrow \mathcal{A}$ be two mappings with $g(0)=f(0)=0$ for which there exists a function $\varphi: \mathcal{A}^{8} \rightarrow[0, \infty)$ such that 


$$
\begin{aligned}
& \left\|h(a x+b y)+h(a x-b y)-2 a^{2} h(x)-2 b^{2} h(y)\right\| \\
& +\left\|f([u v w])-\left[f(u) v^{2} w^{2}\right]-\left[u^{2} f(v) w^{2}\right]-\left[u^{2} v^{2} f(w)\right]\right\| \\
& +\left\|g([r s t])-\left[g(r) s^{2} t^{2}\right]-\left[r^{2} f(x) t^{2}\right]-\left[r^{2} s^{2} f(t)\right]\right\| \\
& \leq \varphi(x, y, u, v, w, r, s, t)
\end{aligned}
$$

for all $h \in\{f, g\}, x, y, u, v, w, r, s, t \in \mathcal{A}$ and nonzero fixed integers $a, b$. Suppose that there exists $L<1$ such that

$$
\varphi(x, y, u, v, w, r, s, t) \leq|4|^{\ell(\ell+2)} L \varphi\left(\frac{x}{2^{\ell^{\ell}}}, \frac{y}{2^{\ell^{\prime}}}, \frac{u}{2^{\ell^{\prime}}}, \frac{v}{2^{\ell^{\prime}}}, \frac{w}{2^{\ell^{\prime}}}, \frac{r}{2^{\ell^{\prime}}}, \frac{s}{2^{\ell^{\prime}}}, \frac{t}{2^{\ell}}\right)
$$

for all $x, y, u, v, w, r, s, t \in \mathcal{A}$. Then there exist a unique quadratic ternary derivation $d: \mathcal{A} \rightarrow \mathcal{A}$ and a unique generalized quadratic ternary derivation $D: \mathcal{A} \rightarrow \mathcal{A}$ (respected to $d$ ) such that

$$
\max \{\|g(x)-D(x)\|,\|f(x)-d(x)\|\} \leq \frac{L^{\frac{1-\ell}{2}}}{|4|} \psi(x)
$$

for all $x \in \mathcal{A}$, where

$$
\begin{gathered}
\psi(x): \max \left\{\varphi\left(\frac{x}{a}, \frac{x}{b}, 0,0,0,0,0,0\right), \varphi\left(\frac{x}{a}, 0,0,0,0,0,0,0\right), \frac{1}{\left|2 b^{2}\right|} \varphi(x, x, 0,0,0,0,0,0),\right. \\
\left.\frac{1}{\left|2 b^{2}\right|} \varphi(x,-x, 0,0,0,0,0,0), \varphi\left(0, \frac{x}{b}, 0,0,0,0,0,0\right)\right\}
\end{gathered}
$$

for all $x \in \mathcal{A}$.

Proof. By (2.2), one can show that

$$
\lim _{n \rightarrow \infty} \frac{1}{|4|^{\ell(\ell+2) n}} \varphi\left(2^{\ell n} x, 2^{\ell n} y, 2^{\ell n} u, 2^{\ell n} v, 2^{\ell n} w, 2^{\ell n} r, 2^{\ell n} s, 2^{\ell n} t\right)=0
$$

for all $x, y, u, v, w, r, s, t \in \mathcal{A}$. Putting $h=g$ in (2.1) and letting $u=v=w=r=s=t=$ 0 in (2.1), we get

$$
\left\|g(a x+b y)+g(a x-b y)-2 a^{2} g(x)-2 b^{2} g(y)\right\| \leq \varphi(x, y, 0,0,0,0,0,0)
$$

for all $x, y \in \mathcal{A}$. Putting $y=0$ in (2.5), we get

$$
\left\|2 g(a x)-2 a^{2} g(x)\right\| \leq \varphi(x, 0,0,0,0,0,0,0)
$$

for all $x \in \mathcal{A}$. Setting $y=-y$ in (2.5), we get

$$
\left\|g(a x-b y)+g(a x+b y)-2 a^{2} g(x)-2 b^{2} g(-y)\right\| \leq \varphi(x,-y, 0,0,0,0,0,0)
$$

for all $x, y \in \mathcal{A}$. It follows from (2.5) and (2.7) that

$$
\left\|2 b^{2} g(y)-2 b^{2} h(-\gamma)\right\| \leq \max \{\varphi(x, y, 0,0,0,0,0,0), \varphi(x,-y, 0,0,0,0,0,0)\}
$$

for all $x, y \in \mathcal{A}$. Putting $y=b y$ in (2.8), we get

$$
\|g(b y)-g(-b y)\| \leq \max \left\{\frac{1}{\left|2 b^{2}\right|} \varphi(x, b y, 0,0,0,0,0,0), \frac{1}{\left|2 b^{2}\right|} \varphi(x,-b y, 0,0,0,0,0,0)\right\}
$$


for all $x, y \in \mathcal{A}$. Setting $x=0$ in (2.5), we get

$$
\left\|g(b \gamma)+g(-b \gamma)-2 b^{2} g(\gamma)\right\| \leq \varphi(0, y, 0,0,0,0,0,0)
$$

for all $y \in \mathcal{A}$. It follows from (2.9) and (2.10) that

$$
\begin{aligned}
& \left\|2 g(b y)-2 b^{2} g(y)\right\| \\
\leq & \max \left\{\frac{1}{\left|2 b^{2}\right|} \varphi(x, b y, 0,0), \frac{1}{\left|2 b^{2}\right|} \varphi(x,-b y, 0,0,0,0,0,0), \varphi(0, y, 0,0,0,0,0,0)\right\}
\end{aligned}
$$

for all $x, y \in \mathcal{A}$. Replacing $x$ and $y$ by $\frac{x}{a}$ and $\frac{x}{b}$ in (2.5), respectively, we get

$$
\left\|g(2 x)-2 a^{2} g\left(\frac{x}{a}\right)-2 b^{2} g\left(\frac{x}{b}\right)\right\| \leq \varphi\left(\frac{x}{a}, \frac{x}{b}, 0,0,0,0,0,0\right)
$$

for all $x \in \mathcal{A}$. Setting $x=\frac{x}{a}$ in (2.6), we get

$$
\left\|2 a^{2} g\left(\frac{x}{a}\right)-2 g(x)\right\| \leq \varphi\left(\frac{x}{a}, 0,0,0,0,0,0,0\right)
$$

for all $x \in \mathcal{A}$. Putting $y=\frac{x}{b}$ in (2.11), we get

$$
\begin{aligned}
& \left\|2 b^{2} g\left(\frac{x}{b}\right)-2 g(x)\right\| \\
\leq & \max \left\{\frac{1}{\left|2 b^{2}\right|} \varphi(x, x, 0,0,0,0,0,0), \frac{1}{\left|2 b^{2}\right|} \varphi(x,-x, 0,0,0,0,0,0), \varphi\left(0, \frac{x}{b}, 0,0,0,0,0,0\right)\right\}
\end{aligned}
$$

for all $x \in \mathcal{A}$. It follows from (2.12), (2.13) and (2.14) that

$$
\|g(2 x)-4 g(x)\| \leq \psi(x)
$$

for all $x \in \mathcal{A}$. Let $\Omega:=\left\{g^{\prime} \mid g^{\prime}: \mathcal{A} \rightarrow \mathcal{A}, g^{\prime}(0)=0\right\}$. For every $g^{\prime}, h^{\prime} \in \Omega$, define

$$
\rho\left(g^{\prime}, h^{\prime}\right):=\inf \left\{C \in(0, \infty):\left\|g^{\prime}(x)-h^{\prime}(x)\right\| \leq C \psi(x), \forall x \in \mathcal{A}\right\} .
$$

It is easy to show that $\rho$ is a complete generalized non-Archimedean metric on $\Omega$ (see $[30,31,34])$. We define $J: \Omega \rightarrow \Omega$ by $J\left(g^{\prime}\right)(x)=\frac{1}{4^{\ell}} g^{\prime}\left(2^{\ell} x\right)$ for all $x \in \mathcal{A}$ and all $g^{\prime}$ $\in \Omega$. One can show that

$$
\rho\left(J g^{\prime}, J h^{\prime}\right) \leq L \rho\left(g^{\prime}, h^{\prime}\right) .
$$

Hence $J$ is a strictly contractive mapping on $\Omega$ with Lipschitz constant $L$. It follows from Theorem 2.1 that $J$ has a unique fixed point $D: \mathcal{A} \rightarrow \mathcal{A}$ in the set $\Lambda=\left\{g^{\prime} \in \Omega\right.$ : $\rho\left(g, g^{\prime}\right)<\infty$, where $d$ is defined by

$$
D(x)=\lim _{n \rightarrow \infty} J^{n} g(x)=\lim _{n \rightarrow \infty} \frac{1}{4^{\ell n}} g\left(2^{\ell n} x\right)
$$

for all $x \in \mathcal{A}$. It follows from (2.4) and (2.15) that

$$
\begin{aligned}
& \left\|D(a x+b y)+D(a x-b y)-2 a^{2} D(x)-2 b^{2} D(y)\right\| \\
& =\lim _{n \rightarrow \infty} \frac{1}{|4|^{\ell n}}\left\|g\left(2^{\ell n} a x+2^{\ell n} b y\right)+g\left(2^{\ell n} a x-2^{\ell n} b y\right)-2 a^{2} g\left(2^{\ell n} x\right)-2 b^{2} g\left(2^{\ell n} y\right)\right\| \\
& \leq \lim _{n \rightarrow \infty} \frac{1}{|4|^{\ell n}} \varphi\left(2^{\ell n} x, 2^{\ell n} y, 0,0,0,0,0,0\right) \\
& \leq \lim _{n \rightarrow \infty} \frac{1}{|4|^{\ell n(\ell+2)}} \varphi\left(2^{\ell n} x, 2^{\ell n} y, 0,0,0,0,0,0\right)=0
\end{aligned}
$$


for all $x, y \in \mathcal{A}$. This shows that $D$ is quadratic.

If $D^{\prime}: \mathcal{A} \rightarrow \mathcal{A}$ is another quadratic mapping which satisfies (2.3), then $D^{\prime}$ is a fixed point of $J$ in $\Lambda$. The uniqueness of the fixed point of $J$ in $\Lambda$ implies that $D=D^{\prime}$. Putting $h=f, u=v=w=r=s=t=0$ in (2.4), we get

$$
\left\|f(a x+b y)+f(a x-b y)-2 a^{2} f(x)-2 b^{2} f(y)\right\| \leq \varphi(x, y, 0,0,0,0,0,0)
$$

for all $x, y \in \mathcal{A}$. By the same reasoning as above, we can show that the limit

$$
d(x)=: \lim _{n \rightarrow \infty} \frac{1}{4^{\ell n}} f\left(2^{\ell n} x\right)
$$

exists for all $x \in \mathcal{A}$. Moreover, we can show that $d$ is a unique quadratic mapping on $\mathcal{A}$ satisfying (2.3).

On the other hand, we have

$$
\begin{aligned}
& \|d([u v w])-[d(u) v w]-[u d(v) w]-[u v d(w)]\| \\
& =\lim _{n \rightarrow \infty} \frac{1}{|4|^{\ell \ell n}}\left\|f\left(4^{\ell n}[u v w]\right)-\left[f\left(2^{\ell n} u\right) v w\right]-\left[u f\left(2^{\ell n} v\right) w\right]-\left[u v f\left(2^{\ell n} w\right)\right]\right\| \\
& \leq \lim _{n \rightarrow \infty} \frac{1}{|4|^{2 \ell n}} \varphi\left(0,0,2^{\ell n} u, 2^{\ell n} v, 2^{\ell n} w, 0,0,0\right) \\
& \leq \lim _{n \rightarrow \infty} \frac{1}{|4|^{\ell(\ell+2) n}} \varphi\left(0,0,2^{\ell n} u, 2^{\ell n} v, 2^{\ell n} w, 0,0,0\right)=0
\end{aligned}
$$

for all $u, v, w \in \mathcal{A}$. Therefore, $d$ is a quadratic ternary derivation on $\mathcal{A}$. Also, we have

$$
\begin{aligned}
& \|D([r s t])-[D(r) s t]-[r d(s) t]-[r s d(t)]\| \\
& =\lim _{n \rightarrow \infty} \frac{1}{|4|^{2 \ell n}}\left\|g\left(4^{\ell n}[r s t]\right)-\left[g\left(2^{\ell n} r\right) s t\right]-\left[r f\left(2^{\ell n} s\right) t\right]-\left[r s f\left(2^{\ell n} t\right)\right]\right\| \\
& \leq \lim _{n \rightarrow \infty} \frac{1}{|4|^{2 \ell n}} \varphi\left(0,0,0,0,0,2^{\ell n} r, 2^{\ell n} s, 2^{\ell n} t\right) \\
& \leq \lim _{n \rightarrow \infty} \frac{1}{|4|^{\ell(\ell+2) n}} \varphi\left(0,0,0,0,0,2^{\ell n} r, 2^{\ell n} s, 2^{\ell n} t\right)=0
\end{aligned}
$$

for all $r, s, t \in \mathcal{A}$. It follows that $D$ is a generalized quadratic ternary derivation (related to $d$ ) on $\mathcal{A}$. This completes the proof.

From now on, we use the following abbreviation for any mappings $g, f: \mathcal{A} \rightarrow \mathcal{A}$ :

$$
\begin{aligned}
\Delta(g, f)\left(x_{1}, \cdots, x_{8}\right) & :=\left\|f\left(a x_{1}+b x_{2}\right)+f\left(a x_{1}-b x_{2}\right)-2 a^{2} h\left(x_{1}\right)-2 b^{2} f\left(x_{2}\right)\right\| \\
& +\left\|g\left(a x_{1}+b x_{2}\right)+g\left(a x_{1}-b x_{2}\right)-2 a^{2} g\left(x_{1}\right)-2 b^{2} g\left(x_{2}\right)\right\| \\
& +\left\|f\left(\left[x_{3} x_{4} x_{5}\right]\right)-\left[f\left(x_{3} x_{4}^{2} x_{5}^{2}\right)\right]-\left[x_{3}^{2} f\left(x_{4}\right) x_{5}^{2}\right]-\left[x_{3}^{2} x_{4}^{2} f\left(x_{5}\right)\right]\right\| \\
& +\left\|g\left(\left[x_{6} x_{7} x_{8}\right]\right)-\left[g\left(x_{6}\right) x_{7}^{2} x_{8}^{2}\right]-\left[x_{6}^{2} f\left(x_{7}\right) x_{8}^{2}\right]-\left[x_{6}^{2} x_{7}^{2} f\left(x_{8}\right)\right]\right\| .
\end{aligned}
$$

Remark. Let $\mathbb{K}=\mathbb{Q}_{2}$ be the 2-adic number field. Let $\mathcal{A}$ be a non-Archimedean Banach algebra on $\mathbb{K}$. Let $\varepsilon$ be a nonnegative real number and let $s$ be a real number such that $s>6$ if $\ell=1$ and $0<s<2$ if $\ell=-1$. Suppose that the mappings $g, f: \mathcal{A} \rightarrow \mathcal{A}$ satisfy $g(0)=f(0)=0$ and

$$
\Delta(g, f)\left(x_{1}, \cdots, x_{8}\right) \leq \varepsilon \max \left\{\left\|x_{i}\right\|^{s}: 1 \leq i \leq 8\right\}
$$


for all $x_{1}, x_{2}, \cdots, x_{8} \in \mathcal{A}$. Then there exist a unique quadratic ternary derivation $d: \mathcal{A} \rightarrow \mathcal{A}$ and a unique generalized quadratic ternary derivation $D: \mathcal{A} \rightarrow \mathcal{A}$ (respected to $d$ ) such that

$$
\begin{aligned}
& \max \{\|g(x)-D(x)\|, \mid f f(x)-d(x) \|\} \\
& \quad \leq|2|^{\frac{\ell(4-s)+s}{2}} \varepsilon\|x\|^{s}\left\{\begin{array}{cl}
2, & \operatorname{gcd}(a, 2)=\operatorname{gcd}(b, 2)=1 ; \\
\max \left\{2^{i s}, 2\right\}, & a=k 2^{i}, \operatorname{gcd}(b, 2)=1 ; \\
\max \left\{2^{j}, 2^{2 j+1}\right\} & \operatorname{gcd}(a, 2)=1, b=m 2^{j} \vee v a=k 2^{i}, b=m 2^{j}(j \geq i) ; \\
\max \left\{2^{i s}, 2^{2 j+1}\right\}, & a=k 2^{i}, \quad b=m 2^{j}(i \geq j) ;
\end{array}\right.
\end{aligned}
$$

for all $x \in \mathcal{A}$, where $i, j, k, m \geq 1$ are integers and $\operatorname{gcd}(k, 2)=\operatorname{gcd}(m, 2)=1$.

Now, we have the following result on superstability of generalized quadratic ternary derivations on non-Archimedean ternary Banach algebras:

Corollary 2.3. Let $p>0$ be a nonnegative real number such that $|2|^{(2 \ell+4) p} \geq 1$ and let $j \in\{3,4, \ldots, 8\}$ be fixed. Suppose that the mappings $g, f: \mathcal{A} \rightarrow \mathcal{A}$ satisfy $g(0)=f(0)$ $=0$ and

$$
\Delta(g, f)\left(x_{1}, \ldots, x_{8}\right) \leq\left(\sum_{i=1}^{8}\left\|x_{i}\right\|^{p}\right)\left\|x_{j}\right\|^{p}
$$

for all $x_{1}, \cdots, x_{8} \in \mathcal{A}$, where $a, b$ are positive fixed integers. Then $f$ is a quadratic ternary derivation and $g$ is a generalized quadratic ternary derivation related to $f$.

Proof. It follows from Theorem 2.2 by taking

$$
\varphi\left(x_{1}, x_{2}, \cdots, x_{8}\right)=\left(\sum_{i=1}^{8}\left\|x_{i}\right\|^{p}\right)\left\|x_{j}\right\|^{p}
$$

for all $x_{1}, \cdots, x_{8} \in \mathcal{A}$ and putting $L=|2|^{-(2 \ell+4) p}$.

\section{Acknowledgements}

C. Park was supported by Basic Science Research Program through the National Research Foundation of Korea funded by the Ministry of Education, Science and Technology (NRF-2012R1A1A2004299). Y.J. Cho was supported by Basic Science Research Program through the National Research Foundation of Korea funded by the Ministry of Education, Science and Technology (NRF-2011-0021821).

\section{Author details}

${ }^{1}$ Department of Mathematics, Research Institute for Natural Sciences, Hanyang University, Seoul 133-791, Korea ${ }^{2}$ Department of Mathematics, Semnan University, P.O. Box 35195-363, Semnan, Iran ${ }^{3}$ Department of Mathematics Education and the RINS, Gyeongsang National University, Chinju 660-701, Korea

\section{Authors' contributions}

All authors conceived of the study, participated in its design and coordination, drafted the manuscript, participated in the sequence alignment, and read and approved the final manuscript.

\section{Competing interests}

The authors declare that they have no competing interests.

Received: 23 October 2011 Accepted: 13 June 2012 Published: 13 June 2012

\section{References}

1. Ulam, SM: Problems in Modern Mathematics. Science ed. Wiley, New York (1940)

2. Hyers, DH: On the stability of the linear functional equation. Proc Natl Acad Sci USA. 27, 222-224 (1941). doi:10.1073/ pnas.27.4.222

3. Rassias, TM: On the stability of the linear mapping in Banach spaces. Proc Amer Math Soc. 72, 297-300 (1978). doi:10.1090/S0002-9939-1978-0507327-1

4. Găvruta, P: A generalization of the Hyers-Ulam-Rassias stability of approximately additive mappings. J Math Anal Appl. 184, 431-436 (1994). doi:10.1006/jmaa.1994.1211 
5. Bourgin, DG: Approximately isometric and multiplicative transformations on continuous function rings. Duke Math J. 16, 385-397 (1949). doi:10.1215/S0012-7094-49-01639-7

6. Badora, R: On approximate ring homomorphisms. J Math Anal Appl. 276, 589-597 (2002). doi:10.1016/50022-247X(02) 00293-7

7. Šemrl, P: The functional equation of multiplicative derivation is superstable on standard operator algebras. Integral Equations Operator Theory. 18, 118-122 (1994). doi:10.1007/BF01225216

8. Badora, R: On approximate derivations. Math Inequal Appl. 9, 167-173 (2006)

9. Cho, Y, Saadati, R, Vahidi, J: Approximation of homomorphisms and derivations on non-Archimedean Lie $C^{*}$-algebras via fixed point method. Discrete Dynamics in Nature and Society 2012, 9 (2012). Article ID 373904

10. Park, C: On an approximate automorphism on a C*-algebra. Proc Amer Math Soc. 132, 1739-1745 (2004). doi:10.1090/ S0002-9939-03-07252-6

11. Park, C, Hou, J, Oh, S: Homomorphisms between Lie JC-algebras and Lie $C^{*}$-algebra. Acta Math Sin. 21, 1391-1398 (2005). doi:10.1007/s10114-005-0629-y

12. Park, C, Rassias, JM: Stability of the Jensen type functional equation in $C^{*}$-algebras: a fixed point approach. Abstr Appl Anal 2009, 17 (2009). Article ID 360432

13. Park, C, Rassias, TM: Homomorphisms in C*-ternary algebras and JB*-triples. J Math Anal Appl. 337, 13-20 (2008). doi:10.1016/j.jmaa.2007.03.073

14. Park, C, Rassias, TM: Homomorphisms and derivations in proper JCQ*-triples. J Math Anal Appl. 337, 1404-1414 (2008). doi:10.1016/j.jmaa.2007.04.063

15. Haag, R, Kastler, D: An algebraic approach to quantum field theory. J Math Phys. 5, $848-861$ (1964). doi:10.1063/ 1.1704187

16. Bagarello, F, Morchio, G: Dynamics of mean-field spin models from basic results in abstract differential equations. J Stat Phys. 66, 849-866 (1992). doi:10.1007/BF01055705

17. Murphy, GJ: Non-Archimedean Banach Algebras. (Thesis) University of Cambridge (1977)

18. Narici, L: Non-Archimedian Banach spaces and algebras. Arch Math (Basel). 19, 428-435 (1968). doi:10.1007/BF01898426

19. Shilkret, N: Non-Archimedian Banach Algebras. Thesis (Ph.D.), Polytechnic Univ., Pro-Quest LLC (1968)

20. Arriola, LM, Beyer, WA: Stability of the Cauchy functional equation over $p$-adic fields. Real Analysis Exchange. 31, 125-132 (2005)

21. Cho, Y, Park, C, Saadati, R: Functional inequalities in non-Archimedean Banach spaces. Appl Math Lett. 10, 1238-1242 (2010)

22. Cho, Y, Saadati, R: Lattictic non-Archimedean random stability of ACQ-functional equation. Adv Difference Equ 2011, 12 (2011). Article No. 31. doi:10.1186/1687-1847-2011-12

23. Eshaghi Gordji, M, Khodaei, H, Khodabakhsh, R: General quartic-cubic-quadratic functional equation in nonArchimedean normed spaces. UPB Sci Bull (Series A). 72, 69-84 (2010)

24. Eshaghi Gordji, M, Savadkouhi, MB: Stability of a mixed type cubic-quartic functional equation in non-Archimedean spaces. Appl Math Lett. 23, 1198-1202 (2010). doi:10.1016/j.aml.2010.05.011

25. Najati, A, Moradlou, F: Hyers-Ulam-Rassias stability of the Aopollonius type quadratic mapping in non-Archimedean spaces. Tamsui Oxford J Math Sci. 24, 367-380 (2008)

26. Saadati, $R, C h o, Y$, Vahidi, J: The stability of the quartic functional equation in various spaces. Comput Math Appl. 60 , 1994-2002 (2010). doi:10.1016/j.camwa.2010.07.034

27. Saadati, R, Park, C: Non-Archimedean $\mathcal{L}$-fuzzy normed spaces and stability of functional equations. Comput Math Appl. 60, 2488-2496 (2010). doi:10.1016/j.camwa.2010.08.055

28. Radu, V: The fixed point alternative and the stability of functional equations. Fixed Point Theory. 4, 91-96 (2003)

29. Diaz, JB, Margolis, B: A fixed point theorem of the alternative for contractions on the generalized complete metric space. Bull Amer Math Soc. 126, 305-309 (1968)

30. Cădariu, L, Radu, V: Fixed points and the stability of Jensen's functional equation. J Inequal Pure Appl Math 4, 7 (2003). no. 1, Article ID 4

31. Cădariu, L, Radu, V: On the stability of the Cauchy functional equation: a fixed point approach. Grazer Math Ber. 346, 43-52 (2004)

32. Cădariu, L, Radu, V: Fixed point methods for the generalized stability of functional equations in a single variable. Fixed Point Theory Appl 2008, 15 (2008). Article ID 749392

33. Jung, S, Kim, T: A fixed point approach to the stability of the cubic functional equation. Bol Soc Mat Mexicana. 12, 51-57 (2006)

34. Mirmostafaee, AK: Hyers-Ulam stability of cubic mappings in non-Archimedean normed spaces. Kyungpook Math J. 50 , 315-327 (2010). doi:10.5666/KMJ.2010.50.2.315

35. Eshaghi Gordji, M, Khodaei, $\mathrm{H}$ : On the generalized Hyers-Ulam-Rassias stability of quadratic functional equations. Abstr Appl Anal 2009, 11 (2009). Article ID 923476

36. Eshaghi Gordji, M: Nearly ring homomorphisms and nearly ring derivations on non-Archimedean Banach algebras. Abstr Appl Anal 2010, 12 (2010). Article ID 393247

37. Eshaghi Gordji, M, Alizadeh, Z: Stability and superstability of ring homomorphisms on non-Archimedean Banach algebra. Abstr Appl Anal 2011, 10 (2011). Article ID 123656

38. Hensel, K: Uber eine neue Begrundung der Theorie der algebraischen Zahlen. Jahresber Deutsch Math Verein. 6, 83-88 (1897)

39. Khrennikov, A: p-adic Valued Distributions in Mathematical Physics. Kluwer Academic Publishers, Dordrecht (1994)

40. Khrennikov, A: Non-Archimedean Analysis: Quantum Paradoxes, Dynamical Systems and Biological Models. Kluwer Academic Publishers, Dordrecht (1997)

41. Gouvêa, FQ: $p$-adic Numbers. Springer-Verlag, Berlin (1997)

42. Vladimirov, VS, Volovich, IV, Zelenov, El: p-Adic Analysis and Mathematical Physics. World Scientific (1994)

doi:10.1186/1687-1812-2012-97

Cite this article as: Park et al: Stability and superstability of generalized quadratic ternary derivations on nonArchimedean ternary Banach algebras: a fixed point approach. Fixed Point Theory and Applications 2012 2012:97. 